\title{
DESIGN OF NANOPOROUS SUPERALLOY MEMBRANES BY SELF-ASSEMBLY OF THE '-PHASE
}

\author{
Joachim Rösler, Oliver Näth, Fabian Schmitz, Debashis Mukherji ${ }^{1}$ \\ Technical University Braunschweig, D-38106 Braunschweig, Langer Kamp 8, Germany
}

Keywords: Superalloys, nanomaterials, porous metals, functional applications

\begin{abstract}
Ni-based superalloys are traditionally used as structural materials in high temperature application. Here we show a new field of application where the self-assembly of the $\gamma^{\prime}$-phase is utilized to produce nanoporous superalloy membranes with particularly interesting functional properties. We describe the manufacturing process, encompassing thermomechanical processing and electrochemical leaching, the resulting microstructure and properties as well as potential areas of application for this new material class.
\end{abstract}

\section{Introduction}

Porous metals with open cell structure have a number of interesting functional applications. They may be used as heat sink material, as substrates for catalytic reactions $[1,2]$ or as permeable membranes for phase separation. In this context, nanoporous materials are of particular interest as they allow for sub-micron phase separation and component miniaturization due to their extreme specific surface area. Unfortunately, it is very difficult to achieve sub-micron porosity using conventional manufacturing methods such as metal powder sintering or casting routes.

An alternative manufacturing process is described here [3]. It utilizes Ni-base superalloys, containing about 40 vol.- $\%$ to 70 vol.- $\%$ of the $\gamma^{\prime}$-phase, which precipitates in a remarkably regular fashion as cube shape particles. The edge length of those cubes is usually a few hundred nanometers. As the cubes are isolated by narrow "channels" of the Ni-matrix, there is no possibility to obtain a nanoporous structure at this state if the matrix is dissolved. However, the $\gamma^{\prime}$-particles grow in a directional manner and connect with each other when the material is stressed at elevated temperatures [4-8]. Result of this well known "rafting" process is the formation of a plate-like $\gamma$ network within the $\gamma$-matrix, whereby both phase become or remain interconnected, respectively, as will be demonstrated below. Once this microstructure consisting of two interpenetrating networks is obtained by the self-assembly of the cubic precipitates, one of the two phases is leached out electrochemically. Consequently, a self-supporting porous structure results, containing open channels with a width of a few hundred nanometers. In this article we describe processing and properties of these novel superalloy membrane materials.

\section{Materials}

Rafting occurs in single crystal as well as polycrystal superalloys. However, the initial orientation of the $\gamma^{\prime}$-cubes changes from grain to grain in the latter case and so does the morphology of the rafted structure after application of a stress at elevated temperatures. Thus, the evolution of the microstructure is less homogeneous in polycrystals so that a less regular porosity results after electrochemical etching. The single crystal alloy CMSX-4 was used throughout this study for this reason unless otherwise stated. The material was solutionized at $1573 \mathrm{~K} / 2,5 \mathrm{~h}+$ $1583 \mathrm{~K} / 6 \mathrm{~h}$, gas fan quenched and precipitation heat treated at $1413 \mathrm{~K} / 6 \mathrm{~h}+1123 \mathrm{~K} / 24 \mathrm{~h}$, leading to the microstructure depicted in fig. 1.

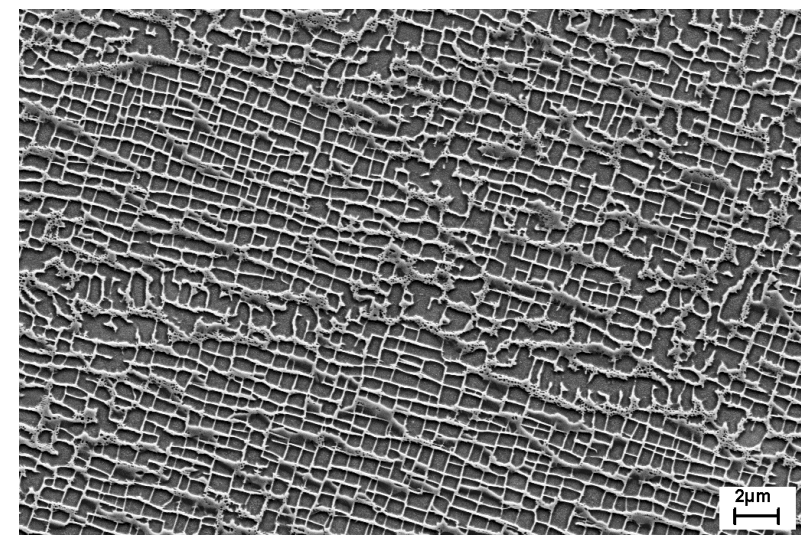

Fig. 1: Microstructure of CMSX-4 after solution and precipitation heat treatment.

\section{Processing}

Processing of nanoporous superalloys requires two basic manufacturing steps: raft formation by thermomechanical treatment and selective leaching of the $\gamma$ or $\gamma^{\prime}$-phase. A tensile stress of $170 \mathrm{MPa}$ oriented parallel to the $<001>$ crystal orientation was applied for $323 \mathrm{~h}$ at $1273 \mathrm{~K}$ to attain the rafted structure shown in fig. 2. As expected, the rafts were oriented perpendicular to the stress axis. The creep deformation led to a plastic strain of $1.01 \%$. The test bars were then cut in longitudinal direction by spark erosion, so that thin sheets, typically $0.2 \mathrm{~mm}$ to $0.5 \mathrm{~mm}$ thick, were obtained.

1 Present address: Institute of Applied Physics, ETH Zürich, HPT Building, CH-8093 Zürich, Switzerland, E-mail: mukherji@jap.phys.ethz.ch. 


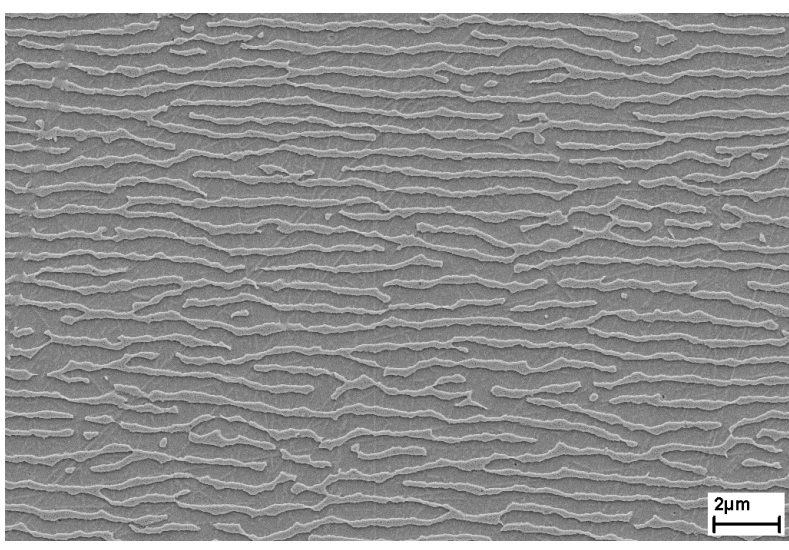

Fig. 2: Rafted microstructure of CMSX-4 after creep deformation at $170 \mathrm{MPa} / 1273 \mathrm{~K} / 323 \mathrm{~h}$.

Selective leaching was performed electrochemically at preselected current density (galvanostatic method) or at preselected potential (potentiostatic method). The experimental setups are shown in fig. 3. For $\gamma$-dissolution, an aqueous electrolyte containing $1 \mathrm{wt} .-\%$ $\left(\mathrm{NH}_{4}\right)_{2} \mathrm{SO}_{4}$ and 1 wt.- $\%$ citric acid was used. A current density of $200 \mathrm{~A} / \mathrm{m}^{2}$ was selected using the galvanostatic method, while a potential of $1.3 \mathrm{~V}$ was used in conjunction with the potentiostatic method. In both cases a duration of $8 \mathrm{~h}$ was sufficient for through thickness etching of sheet thicknesses up to $0.5 \mathrm{~mm}$. The $\gamma^{\prime}$ dissolution was carried out with 1 vol.- $\% \mathrm{HCl}$ in methanol using the potentiostatic method at a potential of $0.3 \mathrm{~V}$. a)

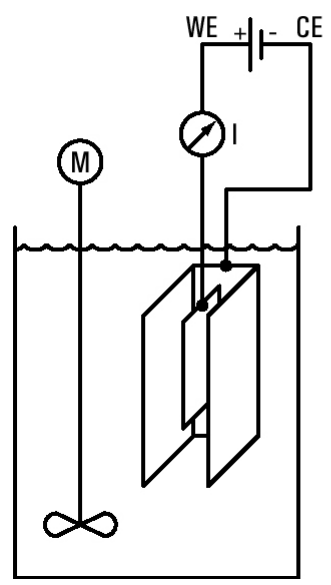

b)

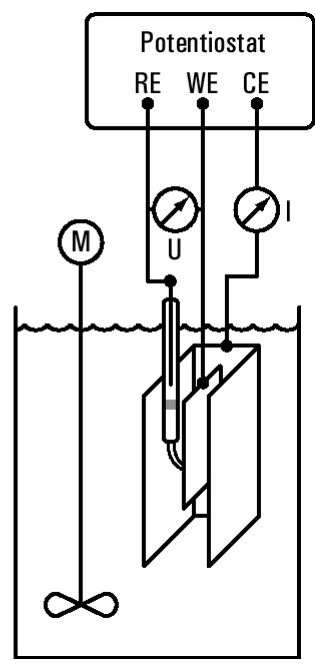

Fig. 3 a,b: Experimental setup used for electrochemical leaching (a: galvanostatic method; b: potentiostatic method; RE: reference electrode; WE: working electrode; CE: counter electrode; M: mixer motor).

\section{Microstructure}

When $\gamma$-dissolution is applied to the as heat treated (but undeformed) material, a loose $\gamma^{\prime}$-powder is obtained [9]. In the case discussed here, the result after electrochemical etching is radically different. Instead of obtaining loose $\gamma^{\prime}$-platelets, a rigid structure results, containing open porosity in the form of channels a few hundred nanometer wide at the location occupied originally by the $\gamma$-matrix (fig. 4). Similarly, a self-supporting structure consisting of the $\gamma$-phase is obtained upon dissolution of the $\gamma^{\prime}$ phase (fig. 5). The pore volume fraction is significantly larger in this case due to the $\gamma^{\prime}$-content of about 70 vol.-\% in CMSX-4.

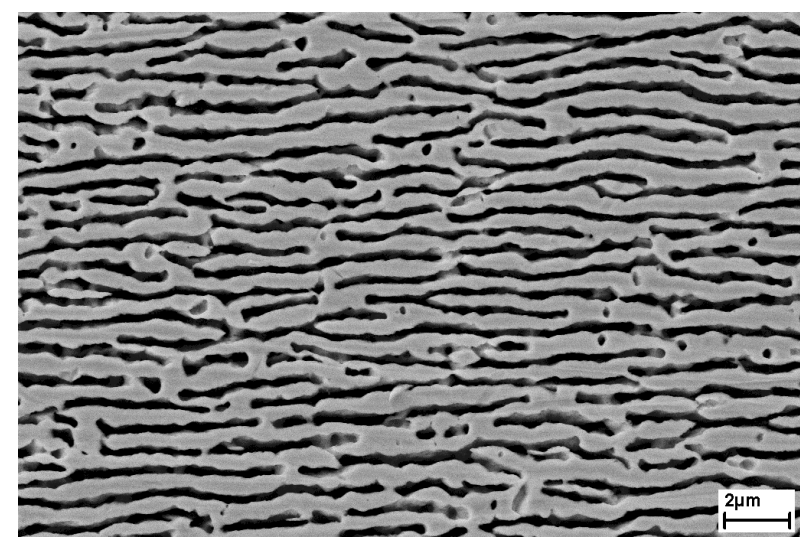

Fig. 4: Microstructure of rafted CMSX-4 after electrochemical leaching of the $\gamma$-phase, using the galvanostatic method. The remaining $\gamma^{\prime}$-phase forms a self-supporting network structure while interconnected porosity is visible at the location of the prior $\gamma$-phase.

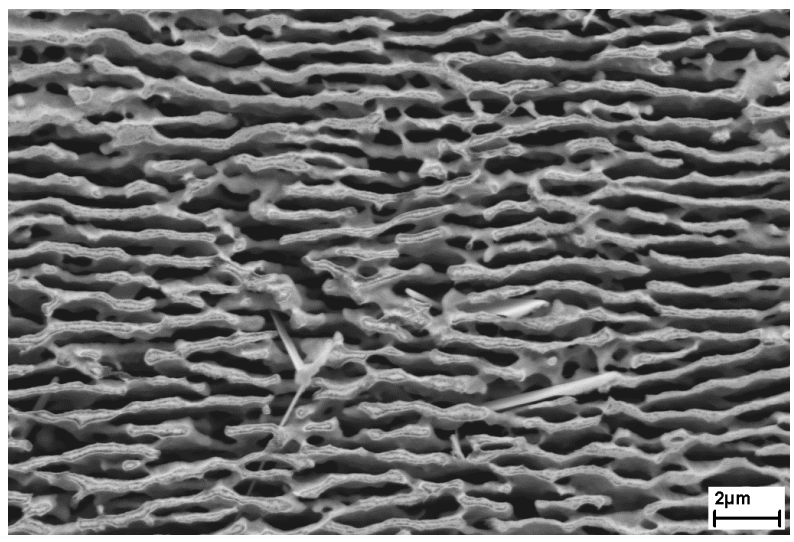

Fig. 5: Microstructure of rafted CMSX-4 after electrochemical leaching of the $\gamma^{\prime}$-phase, using the potentiostatic method. The remaining $\gamma$-phase forms a self-supporting network structure while interconnected porosity is visible at the location of the prior $\gamma^{\prime}$ phase. 
A cross section of an approximately $0.1 \mathrm{~mm}$ thick sheet is shown in fig. 6 after rafting and $\gamma$-dissolution. Note that the porosity is not confined to the surface but is penetrating the entire specimen. This suggests, but does not prove gas permeability. Thus, gas permeability tests were performed as discussed below.

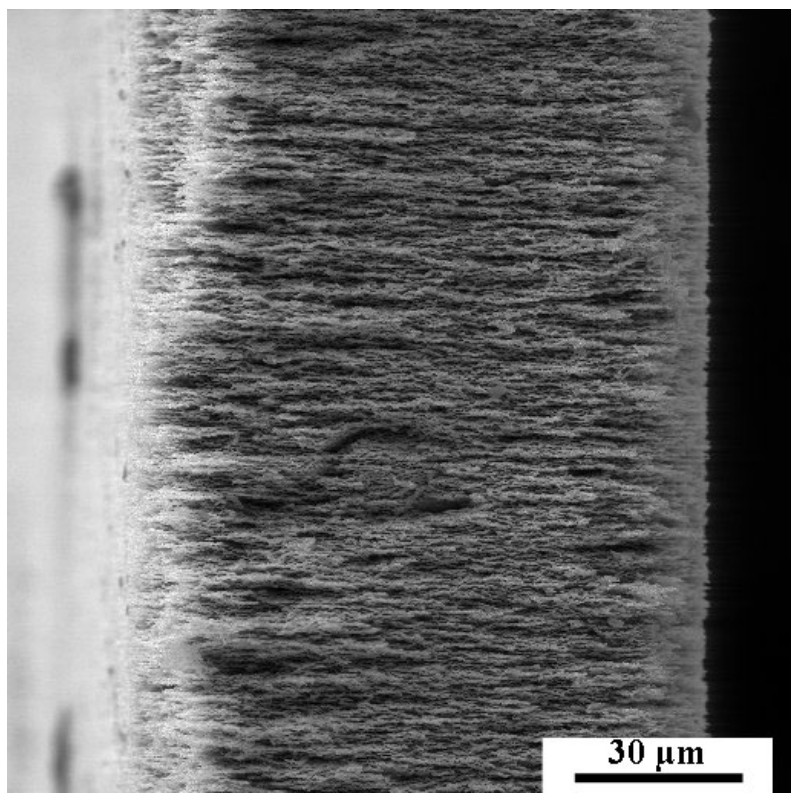

Fig. 6: Cross section of a single crystalline superalloy with composition Ni-4.6Al-6.4Ta-5.7Cr-10.8W-2.1Mo after rafting and electrochemical etching, using an aqueous electrolyte containing $1 \mathrm{wt} .-\%\left(\mathrm{NH}_{4}\right)_{2} \mathrm{SO}_{4}$ and $1 \mathrm{wt} . \%$ citric acid at a current density of $200 \mathrm{~A} / \mathrm{m}^{2}$ for $\gamma$-dissolution (from ref. [3]).

\section{Gas Permeability}

Gas permeability tests using the experimental setup shown in fig. 7 were performed after $\gamma$-dissolution. The porous superalloy samples were placed on a steel holder, containing a central bore hole of cross sectional area A, and sealed using a vacuum-tight plasticine. The outlet side of the steel holder was connected to a container of given volume $\mathrm{V}$, which was evacuated to a pressure of less than $\mathrm{p}_{2 \text {,start }}=300 \mathrm{~Pa}$. A thin plastic film was placed over the superalloy sample at this point in time to inhibit gas flow. The plastic film was subsequently removed, so that an ambient air pressure $\mathrm{p}_{1}$ of about $1.0 \cdot 10^{5} \mathrm{~Pa}$ was applied, and the time $\mathrm{t}$ was monitored until an air pressure $\mathrm{p}_{2, \text { end }}=1.0 \cdot 10^{4} \mathrm{~Pa}$ was reached in the evacuated container. By normalizing the air volume in the container to ambient pressure, the air flow rate under standard condition $\mathrm{F}=\mathrm{V} /(\mathrm{At}) \cdot\left(\mathrm{p}_{2} / \mathrm{p}_{1}\right)$ is obtained. It is plotted as a function of the specimen thickness in fig. 8. As expected, the flow rate increases with decreasing specimen thickness, reaching values as high as $0.12 \mathrm{Nm}^{3} / \mathrm{m}^{2} \mathrm{~s}^{\dagger}$. Consequently, thin sheets are beneficial for two reasons. They lead to improved gas permeability and are more economic to fabricate as the required time for electrochemical etching diminishes.

\footnotetext{
"The symbol "N" expresses that the flow rate is related to standard conditions, i.e. ambient pressure and temperature.
}

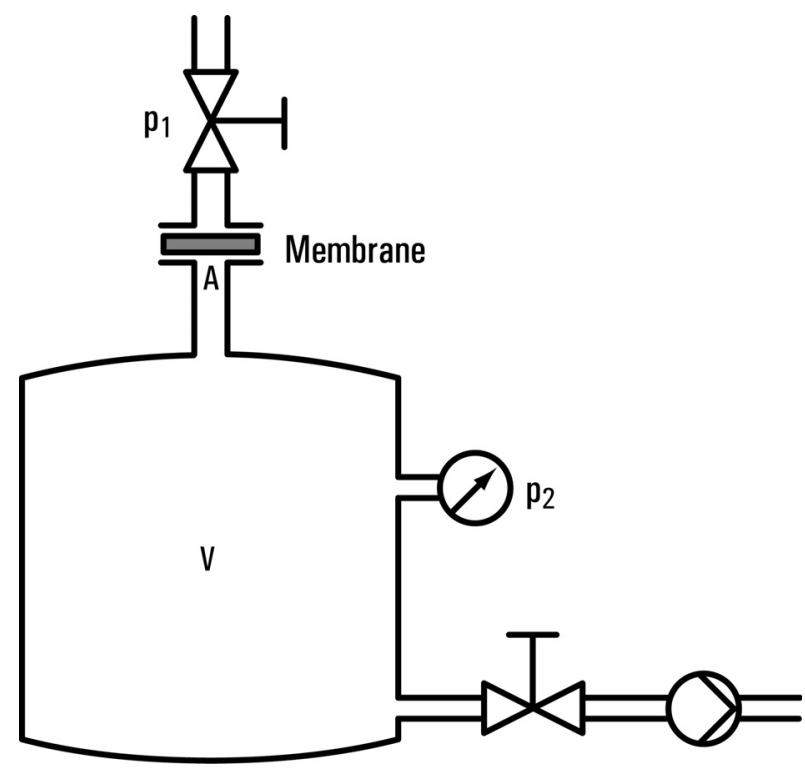

Fig. 7: Experimental setup for measurement of the gas permeability (V: volume of container; A: cross section of central bore hole).

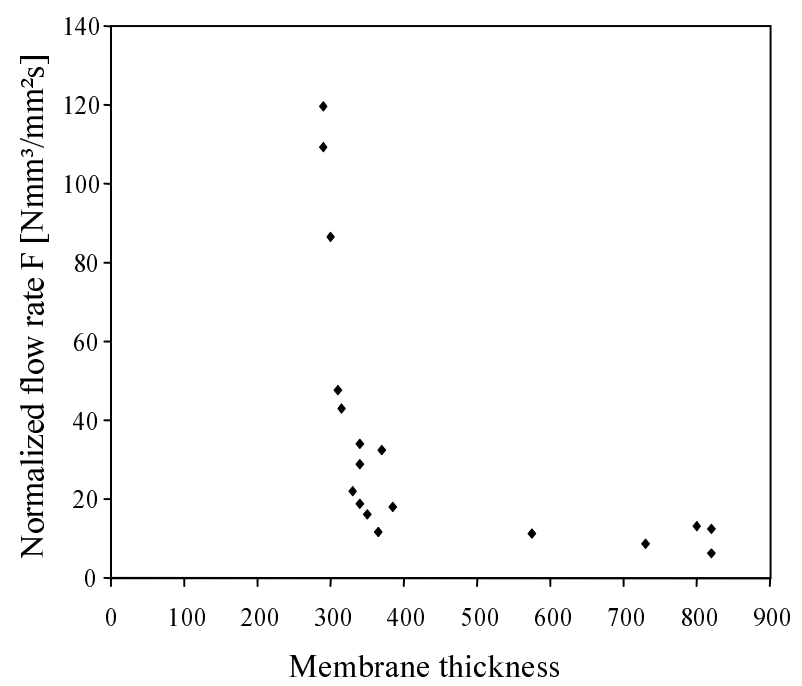

Fig. 8: Normalized flow rate of air through a CMSX-4 membrane as a function of membrane thickness after electrochemical $\gamma$ dissolution.

The gas permeability not only depends on the sheet thickness but also on the pore size and volume fraction. It is anticipated that even higher flow rates are attainable when the $\gamma^{\prime}$-phase instead of the $\gamma$-phase is removed, as this should increase the porosity from approximately 30 vol.- $\%$ to 70 vol.-\%. Furthermore, heat treatment condition and thermomechanical treatment during raft formation will play a role. For instance, longer elevated 
temperature exposure prior to electrochemical etching will lead to a coarser microstructure and thus to larger pores, i.e. higher flow rates. Similarly, the initial $\gamma^{\prime}$-size can be controlled by adjustment of the precipitation heat treatment procedure.

When fluid flow through a channel is considered, one has to distinguish between two limiting cases: continuum flow and molecular flow. Whereas the mean free path of the molecules is much smaller than the width of the channel in the first case, the reverse holds true in the latter case [10]. In other words, molecules are predominantly colliding with each other in case of continuum flow while collisions with the channel walls are more frequent in case of molecular flow. There is an interesting consequence of molecular flow when gas mixtures, containing molecules $\mathrm{A}$ and $\mathrm{B}$ of molecular mass $\mathrm{m}_{\mathrm{A}}$ and $\mathrm{m}_{\mathrm{B}}$, are considered. Given an initial concentration $c_{A}$ and $c_{B}$ of both species, a concentration ratio $\left(\mathrm{c}_{\mathrm{A}} / \mathrm{c}_{\mathrm{B}}\right) \cdot\left(\mathrm{m}_{\mathrm{B}} / \mathrm{m}_{\mathrm{A}}\right)^{1 / 2}$ is obtained after effusion through a narrow hole. Thus, separation of gases with different molecular weight becomes possible. Reason for the separation characteristic is the inverse proportionality between mean molecular velocity and square root of the molecular mass for a gas in equilibrium.

The mean free path of gas molecules at ambient temperature and pressure is of the order of $100 \mathrm{~nm}$, i.e. it is similar to the channel width of the porous superalloy membranes. Thus, the situation is in-between the limiting cases discussed above and the question arises, whether gas separation is possible. To check the separation characteristic of the nanoporous superalloy membranes, a $\mathrm{H}_{2} / \mathrm{CO}_{2}$ gas mixture containing 52 vol.-\% $\mathrm{H}_{2}$ and 48 vol.- $\% \mathrm{CO}_{2}$ was used. It was guided over the membrane at a pressure $\mathrm{p}_{1}=1.0 \cdot 10^{5} \mathrm{~Pa}$ until the pressure $p_{2}$ was raised from less than $300 \mathrm{~Pa}$ to $1.0 \cdot 10^{4} \mathrm{~Pa}$. Afterwards the concentration of the permeated gas mixture was measured by gas chromatography as 64 vol.- $\% \mathrm{H}_{2}$ and 36 vol.- $\% \mathrm{CO}_{2}$. Thus, a noticeable enrichment of the lighter species occurs even though the concentration ratio increases only by a factor 1.64, whereas the square root of the mass ratios $\left(\mathrm{m}_{\mathrm{CO} 2} / \mathrm{m}_{\mathrm{H} 2}\right)^{1 / 2}$ is 4.69 . This is understandable, as the size of the open channels is not small enough to entirely fulfill the requirements for molecular flow. Furthermore, the observed separation characteristic may be a consequence of preferential $\mathrm{CO}_{2}$ adsorption at the superalloy surface. If this were the case, gas separation would be a transient effect and terminate as soon as an equilibrium between adsorption and desorption is achieved. To inspect this possibility, a $\mathrm{CO}_{2}$ flow of $1167 \mathrm{~mm}^{3} / \mathrm{s}$ was guided through the membrane material for $25 \mathrm{~h}$ at ambient temperature and a pressure $\mathrm{p}_{2}=1.0 \cdot 10^{5} \mathrm{~Pa}$. The separation experiment was then repeated and a separation ratio of 1.45 was measured. Thus, it is concluded that adsorption plays a role. However, there is a definite separation effect stemming from the dimensions of the porosity on the nanoscale.

\section{Discussion}

The ability to form a porous solid by selective leaching of the $\gamma$ or $\gamma^{\prime}$-phase demonstrates that both phases must have formed interpenetrating, self-supporting networks during rafting. If one phase would be present as isolated precipitates, the leaching process would either lead to loose powder or it would stop once the precipitates connected to the surface have been removed.

Numerous leaching experiments, not shown here, have been conducted over a wide range of creep strains. They suggest that creep strains of $1 \%$ or even less are sufficient for the two phases to built interpenetrating networks. More specifically, there seems to be a close correlation between this event and the minimum of the creep strain rate, which occurs at around $0.5 \%-1 \%$ plastic strain $[11,24]$. Thus, directional growth of the $\gamma^{\prime}$-phase to the point were the isolated precipitates are linked to a network structure seems to cause strengthening. This can be interpreted in the frame of composite models, showing that load transfer from the softer matrix onto the harder strengthening phase is the more pronounced the higher the aspect ratio of the strengthening phase is [12-16]. Once the precipitates have linked to an interpenetrating network, both phases must deform simultaneously ${ }^{2}$ and the highest possible strength of the composite is reached. Softening occurs from now on due to coarsening of the microstructure.

Porous solids were also successfully produced on ruptured creep samples of the single crystal alloy Ni-4.6Al-6.4Ta-5.7Cr-10.8W2.1Mo after loading at $\mathrm{T}=1273 \mathrm{~K}$ and $\sigma=190 \mathrm{MPa}$ for 145 hours. Its $\gamma^{\prime}$-content is about 55 vol.-\%. The result shows that microstructure inversion, leading to $\gamma$-precipitates embedded in a $\gamma^{\prime}$-matrix, does not necessarily occur during creep life. However, there may be testing conditions not investigated here, were such a structure inversion occurs. Also, it should be noted that the $\gamma^{\prime}$ content of the above alloy is less than that of CMSX-4. Leaching experiments as describe here are a sensitive tool to clarify the nature of the $\gamma^{\prime}$-morphology for any thermomechanical history of interest.

Porous metals can be processed by numerous techniques [17]. Examples are foaming of liquid metal $[18,19]$, eutectic solidification involving a gaseous phase [20], powder metallurgical processing including selective leaching of an element $[21,22]$ and gas phase deposition onto a porous polymer substrate [23]. However, the processing route described here is unique as it produces open porosity with unsurpassed fineness and regularity of the porosity. Consequently, nanoporous superalloys are ideal candidates for a number of applications. They may allow for miniaturization of heat exchangers and chemical reactors due to their very large specific surface area. In this context it is noteworthy that nickel is a good catalyst for a number of chemical reactions, in particular those involving hydrogen adsorption and desorption. They may be used to filter submicron particles such as soot from the exhaust of diesel engines or bacteria in medical applications. The ability to sterilize microorganisms, such as the escherichia coli bacterium shown in fig. 9, by resistance heating of the metallic filter is an added advantage not amenable to ceramic filters or polymer membranes.

The possibility to separate gas molecules of different molecular weight is also of practical interest. $\mathrm{CO}_{2}$ sequestration from $\mathrm{H}_{2} / \mathrm{CO}_{2}$ mixtures obtained by catalytic conversion of hydrocarbon fuels is a much discussed example, as this would enable energy generation from fossil fuels without $\mathrm{CO}_{2}$ emission into the atmosphere. Furthermore, clean $\mathrm{H}_{2}$ fuel is required to power devices such as polymer exchange membrane (PEM) fuel cells. Even though separation is not quantitative, the measured $\mathrm{H}_{2}$ enrichment after flow through the superalloy membrane is

\footnotetext{
${ }^{2}$ Note that deformation of the precipitate phase may start prior to network formation due to load transfer in the course of creep deformation. In other words, network formation of the $\gamma^{\prime}$-phase is a sufficient, but not necessary condition for deformation of this phase to occur in the course of creep loading.
} 
significant and may aid the development of technically viable solutions.

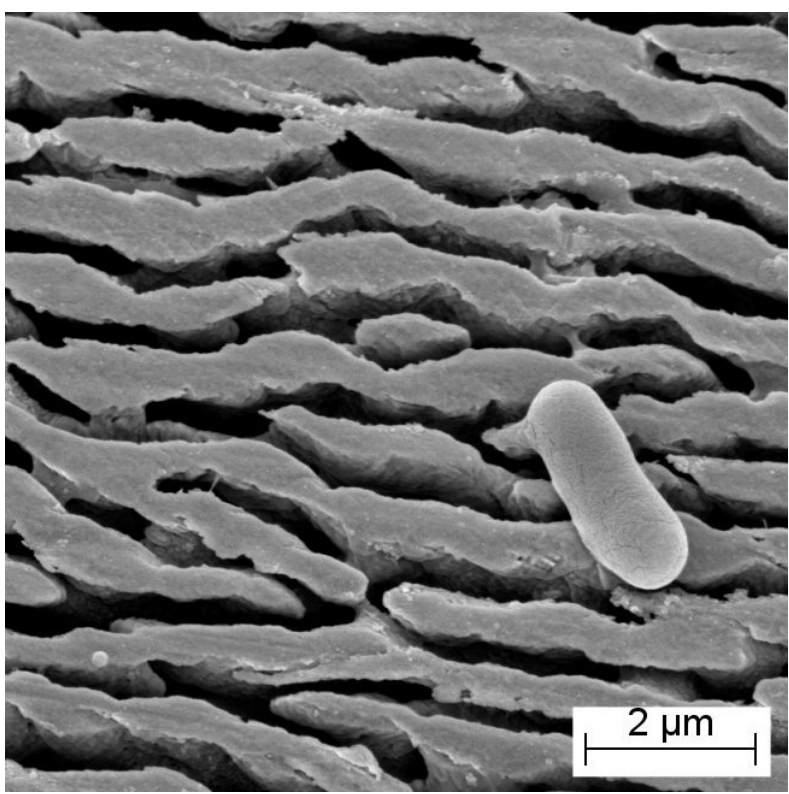

Fig. 9: Escherichia Coli bacterium on top of a nanoporous superalloy membrane (from ref. [3]). The narrow channels ensure quantitative filtration of the microorganisms. The membrane was produced by $\gamma$-dissolution of a superalloy with the following composition: Ni-4.6Al-6.4Ta-5.7Cr-10.8W-2.1Mo.

Nanoporous superalloys can also be used as gas permeable and electrically conductive substrates for thin film deposition. The point here is that the minimum thickness required to produce dense films is directly correlated with the pore dimensions. Hence, the advantage compared to other porous metals lies in the smaller pore dimensions, allowing for a reduced film thickness. For instance, this may enable production of solid oxide fuel cells with diminished thickness of the solid electrolyte membrane, thus reducing the electrical resistance of the cell.

The manufacturing process described here not only allows production of entirely porous sheets. It is also possible to build sequences of porous and non-porous area. Fig. 10a shows a layered structure of a solid core with nanoporous surface areas. Structures like that may be of particular interest in applications were strength is a key issue but gas permeability is not required or even undesired. A lateral sequence of porous and solid areas, obtained by surface masking with a protective varnish prior to etching, is depicted in fig. $10 \mathrm{~b}$.

\section{Summary}

We have demonstrated manufacturing of nanoporous superalloy membranes combining the following set of properties:

- extremely fine porosity, a few hundred nanometers wide

- large pore volume fraction of about $50 \mathrm{vol.- \%} \pm 20$ vol.- $\%$

- regular porosity shape

- gas permeability
- thermal and electrical conductivity

- good oxidation resistance

Key manufacturing steps are (i) formation of self-supporting and interpenetrating networks consisting of the $\gamma$ - and $\gamma^{\prime}$-phase by thermomechanical processing; (ii) selective dissolution of one of the two phases.

The material seems best suited for applications in phase separation, heat transfer and catalysis whenever highest possible specific surface areas in conjunction with metallic properties are required.

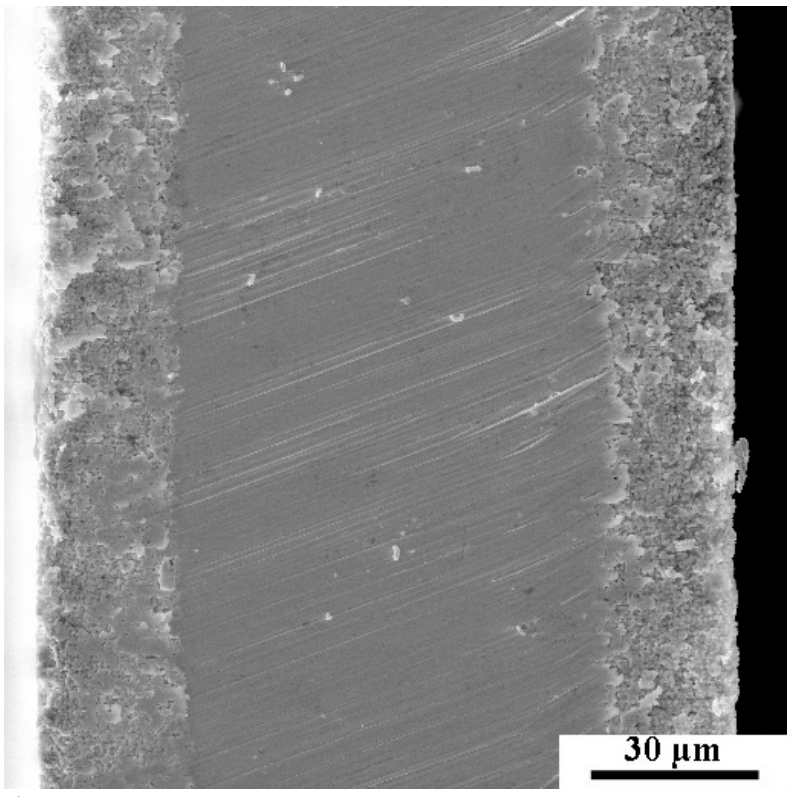

a)

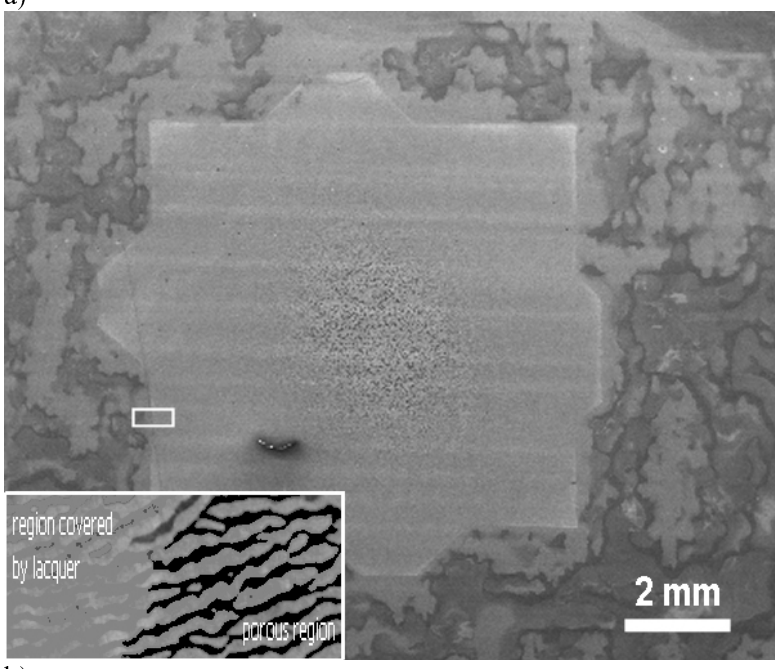

b)

Fig. 10 a,b: Examples for solid/porous sequences obtained by electrochemical etching of a single crystal superalloy with composition Ni-4.6Al-6.4Ta-5.7Cr-10.8W-2.1Mo. A layered structure is shown in (a), while lateral structuring is shown in (b). 


\section{Acknowledgement}

We would like to thank the Deutsche Forschungsgemeinschaft for financial support of this work. The help of Dr. M. Rohde, Gesellschaft für Biologische Forschung, Braunschweig, Germany in preparing the sample with the escherichia coli bacterium is greatly appreciated.

\section{References}

[1] E.N. Gobina, R. Hughes, Membrane Technology 1997, 86, 8.

[2] G. Saracco, H.W.J.P. Neomagus, G.F. Versteeg, W.P.M. van Swaaij, Chemical Engineering Science 1997, 54, 1999.

[3] J. Rösler, D. Mukherji, Adv. Eng. Mat. 2003, 5, 916.

[4] H.-A. Kuhn, H. Biermann, T. Ungar, H. Mughrabi, Acta metall. mater. 1991, 39, 2783.

[5] T.M. Pollock, A.S. Argon, Acta metall. mater. 1994, 42, 1859.

[6] A. Pineau, A., Acta metall. 1976, 24, 559.

[7] M. Veron, Y. Brechet, F. Louchet, Acta mater. 1996, 44, 3633.

[8] W.C. Johnson, M.B. Berkenpas, D.E. Laughlin, Acta metall. 1988, 36, 3149.

[9] E. Kny, Praktische Metallographie 1976, 13, 549.

[10] W.J. Moore, Physical Chemistry, Prentice Hall Inc., New Jersey, p. 139-140 (1972).

[11] D. Mukherji, J. Rösler, Z. Metallkd., 2003, 94, 478.

[12] G. Bao, J.W. Hutchinson, R.M. McMeeking, Acta Metall. Mater., 1991, 39, 1871.

[13] T. Christman, A. Needleman, S. Suresh, Acta Metall., 1989, 37, 3029.

[14] V. Tvergaard, Acta Metall. Mater., 1990, 38, 185.

[15] J. Rösler, Int. J. of Materials \& Product Technology, 2003, 18,70 .

[16] J. Rösler, in Encyclopedia of Materials: Science \& Technology, K.H.J. Buschow, R.W. Cahn, M.C. Flemmings, B. Ilschner, E.J. Kramer, S. Mahajan (eds.), Elsevier Osford, 2001, 2, 1806.

[17] J. Banhart, Prog. Mater. Sci., 2001, 46, 559.

[18] J.C. Elliot, US Patent 2,983,597, 1961.

[19] I. Jin, L.D. Kenny, H. Sang, US Patent 4,973,358, 1990.

[20] V.I. Shapovalov, US Patent, 5,181,549, 1993.

[21] J. Baumeister, German Patent 4,018,360, 1990.

[22] A.B. Stiles, German Patent 2,037,928, 1971.

[23] E. Pinkhasov, US Patent 5,011,638, 1991.

[24] W. Schneider, J. Hammer, H. Mughrabi, in Superalloys 1992, S.D. Antolovich, R.W. Stusrud, R.A. Mackay, D.L. Anton, T. Khan, R.D. Kissinger, D.L. Klarstrom (Eds.), TMS 1992, p. 589. 